Tohoku Math. J.

54 (2002), 85-104

\title{
MEROMORPHIC FIRST INTEGRALS: SOME EXTENSION RESULTS
}

\author{
ROGÉRIO S. MOL \\ (Received April 3, 2000, revised March 29, 2001)
}

\begin{abstract}
We present sufficient conditions of extending a meromorphic function which is defined outside an analytic compact curve in a complex surface. The function we deal with is a first integral for a holomorphic foliation in the whole surface. The key to extension is the study of singularities of the foliation on the complex curve.
\end{abstract}

1. Introduction. We consider a singular holomorphic foliation $\mathcal{F}$ in a complex surface $M$, equipped with a meromorphic first integral defined outside a compact complex curve $S$. We are basically concerned with the following question: under which conditions does $\mathcal{F}$ admit a meromorphic first integral in the entire surface $M$ ?

Proposition 2 asserts that when $S$ is not $\mathcal{F}$-invariant, then the first integral extends to the whole $M$. To study the case where $S$ is $\mathcal{F}$-invariant, some necessary hypotheses are set on the singularities of $\mathcal{F}$ in $S$ : we assume that any singularity contained in $S$ has no saddle-nodes in its desingularization. Such a singularity is called a generalized curve. We have:

THEOREM A. Let $\mathcal{F}$ be a singular holomorphic foliation in a complex surface $M$ admitting a meromorphic first integral $h$ in $M \backslash S$, where $S$ is a compact, smooth, connected complex curve. If some singularity of $\mathcal{F}$ in $S$ is a non-dicritical generalized curve, then $h$ extends to a meromorphic first integral for $\mathcal{F}$ in $M$.

In the case where all singularities in $S$ are dicritical (here, being dicritical means having an infinite number of separatrices), further hypothesis are set on the curve $S$ :

THEOREM B. Let $\mathcal{F}$ be a singular holomorphic foliation in a complex surface $M$ admitting a meromorphic first integral $h$ in $M \backslash S$, where $S$ is a compact, smooth, connected complex curve with negative self-intersection number. If all singularities of $\mathcal{F}$ in $S$ are generalized curves, then $h$ extends to a meromorphic first integral defined in $M$.

When $S$ has non-negative self-intersection number, the extension is still possible if $S$ contains an adequate amount of special dicritical singularities, which we call ordinary dicritical:

THEOREM C. Let $\mathcal{F}$ be a singular holomorphic foliation in a complex surface $M$ admitting a meromorphic first integral h outside a compact, smooth, connected complex curve $S$ with self-intersection number $n \geq 0$. Suppose that the singularities of $\mathcal{F}$ in $S$ are generalized

2000 Mathematics Subjetct Classification. Primary 32S65; Secondary 32A20.

Key words and phrases. Holomorphic foliation, first integral, meromorphic function.

This work was supported by PRPq-Universidade Federal de Minas Gerais and CNPq-Brasil. 
curves. If there are at least $n+1$ ordinary dicritical singularities in $S$, then $h$ extends to a meromorphic first integral in $M$.

The basic tool for the proofs of Theorems A, B and C is Lemma 2, which is called Extension Lemma. It asserts that a meromorphic first integral in a neighborhood of one of the separatrices of a simple singularity extends to a neighborhood of the singularity. This, along with some results on the extension of meromorphic functions, transforms our problem into one of finding separatrices through the desingularization divisor.

Sections 5 and 6 are devoted to the situation where $M$ is a complex projective space $\boldsymbol{C} P^{n}$. We study the problem in dimension two and then show how the problem in $\boldsymbol{C} \boldsymbol{P}^{n}$ reduces to a two-dimensional one.

In Section 7 we give conditions upon that similar extension theorems apply to a foliation by curves in a complex manifold $M$ of dimension $n$. Finally, in Section 8 , we produce variants of Theorems A, B and C where we extend closed meromorphic one-forms defining a foliation in a complex surface. With some adaptations, the techniques of the previous sections also apply to this situation.

This work was developed as a Ph. D. thesis at IMPA, Brazil. I am grateful to my advisor C. Camacho. I also thank P. Sad and B. Azevedo Scárdua for their valuable comments and contributions.

2. Proofs of the main theorems. Let $\mathcal{F}$ be a singular holomorphic foliation defined in a complex surface $M$, that is, a two-dimensional complex manifold. By a singular holomorphic foliation we mean a holomorphic foliation outside an analytic set $s(\mathcal{F})$, the singular set of $\mathcal{F}$, of codimension two or greater. We remark that, as a consequence of Levi's extension theorem, a singular holomorphic foliation of dimension one is induced by a holomorphic vector field in a neighborhood of each point (see [L]). We say that a point $p \in s(\mathcal{F})$ is a reduced singularity if the eigenvalues $\lambda_{1}$ and $\lambda_{2}$ of the linear part of a vector field which defines $\mathcal{F}$ at $p$ satisfy one of the following:

(i) $\lambda_{1} \neq 0, \lambda_{2} \neq 0, \lambda_{2} / \lambda_{1} \notin Q^{+}$;

(ii) $\lambda_{1} \neq 0, \lambda_{2}=0$ or $\lambda_{1} \neq 0, \lambda_{2}=0$.

Singularities of type (i) are said to be simple. The special case in which $\lambda_{1} / \lambda_{2} \in Q^{-}$is called a resonance. Singularities of type (ii) are called saddle-nodes.

A meromorphic function $h$ is called a meromorphic first integral for $\mathcal{F}$ if its indeterminacy set is contained in $s(\mathcal{F})$ and its level curves contain the leaves of $\mathcal{F}$. Simple singularities which admit meromorphic first integrals are linearizable resonances, as the following results prove:

PROPOSITION 1. Let $p$ be a reduced singularity of $\mathcal{F}$ admitting a meromorphic first integral in some neighborhood. Then $p$ is a resonance.

PROOF. Suppose first that $p$ is simple and non resonant. Then $\mathcal{F}$ is formally linearizable (see [CS1]); in formal coordinates at $p, \mathcal{F}$ is given by $\omega_{p}=x d y-\lambda y d x, \lambda \in \boldsymbol{C}-\boldsymbol{Q}$. 
Write

$$
F(x, y)=\sum_{m \geq m_{0}, n \geq n_{0}} a_{m n} x^{m} y^{n}
$$

the development in Laurent series of the (formal) first integral for $\mathcal{F}$. We have

$$
\begin{aligned}
0 & =\omega_{p} \wedge d F \\
& =(x d y-\lambda y d x) \wedge\left(\sum_{m \geq m_{0}, n \geq n_{0}} m a_{m n} x^{m-1} y^{n} d x+\sum_{m \geq m_{0}, n \geq n_{0}} n a_{m n} x^{m} y^{n-1} d y\right) \\
& =-\sum_{m \geq m_{0}, n \geq n_{0}}(m+\lambda n) a_{m n} x^{m} y^{n} d x \wedge d y .
\end{aligned}
$$

Since $\lambda \notin Q$, we must have $a_{m n}=0$ for any $(m, n) \neq(0,0)$, contradicting the fact that $F$ is non-constant.

Similar formal calculations employing Dulac's normal form ([CS1]) show that $p$ cannot be a saddle-node.

We say that a one-dimensional analytic set $S$ is a separatrix through $p \in s(\mathcal{F})$ if $p \in$ $S$ and $S$ is invariant by $\mathcal{F}$. We remark that a simple singularity admits a pair of smooth separatrices. For a saddle node, we can assure the existence of one smooth separatrix (see [CS1]). In general, a singularity always admits at least one separatrix ([CS]).

LEMMA 1 (Linearization lemma). Let $p \in s(\mathcal{F})$ and $S$ a separatrix for $\mathcal{F}$ at $p$. Suppose that $\mathcal{F}$ admits a meromorphic first integral $F$ in a neighborhood $V$ of $S^{*}=S \backslash\{p\}$. Then the holonomy with respect to $S$ is linearizable.

Proof. Let $\gamma:[0,1] \rightarrow S^{*}$ be a simple closed path around $p$. Let $q=\gamma(0)=\gamma(1)$ and $\Sigma$ a small complex disc centered at $q$, contained in $V$ and transversal to $\mathcal{F}$, provided with a complex coordinate $w$. If $h_{\gamma}: \Sigma \rightarrow \Sigma$ is the holonomy map associated to $\gamma$, then $F\left(h_{\gamma}(w)\right)=F(w)$ for any $w \in \Sigma$. Setting a new complex coordinate $z$ in which $\left.F\right|_{\Sigma}$ reads $\left.F\right|_{\Sigma}(z)=z^{n}$, we have $\left(h_{\gamma}(z)\right)^{n}=z^{n}$. Therefore, $h_{\gamma}(z)=e^{2 \pi i k / l} z$, where $k, l \in \boldsymbol{Z}$ and $l \mid n$.

LEMMA 2 (Extension lemma). Let $p \in s(\mathcal{F})$ and $S$ a separatrix for $\mathcal{F}$ at $p$. Suppose that $\mathcal{F}$ admits a meromorphic first integral $F$ in a neighborhood $V$ of $S^{*}=S \backslash\{p\}$. If $p$ is a simple singularity, then $F$ extends meromorphically to a neighborhood of $p$.

ProOf. The previous Lemma and [MM] show that $\mathcal{F}$ is a linearizable resonance at $p$; there exists a system of coordinates $(x, y)$ centered at $p$ such that $\mathcal{F}$ is defined by $\omega=$ $x d y-\lambda y d x, \lambda \in Q^{+}$. Write $\lambda=-p / q, p, q \in N,(p, q)=1$. Suppose that $S$ has the local equation $\{y=0\}$. Developing $F$ in the Laurent series

$$
F(x, y)=\sum_{n \geq n_{0}} a_{m n} x^{m} y^{n},
$$


we have

$$
0=d F \wedge \omega=\sum_{n \geq n_{0}}(m q-n p) a_{m n} x^{m} y^{n} d x \wedge d y .
$$

We see that $a_{m n} \neq 0$ if and only if $m q-n p=0$, which occurs if and only if there exists $l \in \boldsymbol{Z}$ such that $m=l p$ and $n=l q$. It is then possible to rewrite

$$
F(x, y)=\sum_{l \geq l_{0}} a_{l p, l q}\left(x^{p} y^{q}\right)^{l}
$$

for some $l_{0} \in \boldsymbol{Z}$. This shows that $F$ extends meromorphically to a neighborhood of 0 .

Levi's extension theorem, which provides the extension of a meromorphic function defined in a Hartogs' domain to its holomorphic closure ([Siu]), allows us to prove the following:

LEMMA 3. Let M be a complex surface and $S$ a smooth, compact, connected complex curve. Suppose that $h$ is a meromorphic function defined in $M \backslash S$. If $h$ extends as a meromorphic function to $(M \backslash S) \cup V_{p}$, where $V_{p}$ is a neighborhood of a point $p \in S$, then it extends meromorphically to $M$.

ProOf. Let $\mathcal{W}$ be the union of the points $q \in S$ for which there exists a neighborhood $V_{q}, q \in V_{q}$, such that $h$ extends meromorphically to $(M \backslash S) \cup V_{q} . \mathcal{W}$ is non-empty by hypothesis and open from its definition. Let us show that it is closed. Take $p_{0} \in S$ in the closure of $\mathcal{W}$. This means that there exists a sequence $q_{n} \in \mathcal{W}$ such that $q_{n} \rightarrow p_{0}$. Chose a coordinate neighborhood $U_{p_{0}}$ around $p_{0}, \Phi=(x, y): U_{p_{0}} \rightarrow \boldsymbol{C}^{2}$ a coordinate chart, such that $P:=\Phi\left(U_{p_{0}}\right)$ is a polydisc and $\Phi\left(S_{0} \cap U_{p_{0}}\right)=\{y=0\}$. Take $n_{0}$ sufficiently large so that $q_{n_{0}} \in U_{p_{0}}$. Then $P \backslash\{y=0\} \cup \Phi\left(U_{p_{0}} \cap V_{q_{n_{0}}}\right)$ is a Hartogs' domain. Levi's theorem assures that $h$ extends meromorphically to $U_{p_{0}}$. Therefore, $p_{0} \in \mathcal{W}$ and the result follows.

Let $\mathcal{F}$ be a foliation in a complex surface $M$ admitting a meromorphic first integral in $M \backslash S$, where $S$ is a smooth, compact, connected complex curve. We are concerned with finding conditions for extending the meromorphic function to the whole $M$. First of all, if $S$ is not $\mathcal{F}$-invariant, then extension is immediate:

PROPOSITION 2. Let $M$ be a complex surface with a singular holomorphic foliation $\mathcal{F}$ admitting a meromorphic first integral $h$ in $M \backslash S$, where $S$ is a smooth, connected complex curve. If $S$ is not $\mathcal{F}$-invariant, then $h$ extends to $M$ as a meromorphic first integral for $\mathcal{F}$.

Proof. Let $p \in S$ be a regular point of $\mathcal{F}$ where the foliation is transversal to $S$. Choose a coordinate neighborhood $U_{p}$ around $p$ and $\Phi=(x, y): U_{p} \rightarrow \boldsymbol{C}^{2}$ a coordinate chart such that $P:=\Phi\left(U_{p}\right)$ is a polydisc, $\Phi\left(S \cap U_{p}\right)=\{y=0\}$ and $\left.\mathcal{F}\right|_{U_{p}}$ is a foliation with vertical leaves given by $d x=0$. Since $h$ is a first integral, we have that $h(x, y)=h(x)$ for $(x, y) \in P \backslash\{y=0\}$. Therefore, $h$ extends meromorphically to $\{y=0\}$ by setting 
$h(x, 0)=h(x)$. This yields the extension of $h$ to $S$ outside $s(\mathcal{F}) \cap S$ and the points of tangency between $\mathcal{F}$ and $S$. They constitute, however, a codimension two analytic set, and the meromorphic extension to them is straight.

Suppose now that $S$ is invariant by $\mathcal{F}$. From Proposition 1, it is reasonable to assume that the singularities of $\mathcal{F}$ over $S$ do not have saddle-nodes in their desingularization; they are generalized curves, according to the definition in [CLS].

Let $\pi: \tilde{M} \rightarrow M$ be a sequence of blow-ups that desingularizes $s(\mathcal{F}) \cap S$ (see [Sei]). We consider the desingularization divisor $D=\pi^{-1}(S)=\bigcup_{i=0}^{n} P_{i}$, where $P_{0}=\pi^{*}(S)$ is the strict transform of $S$ and $\bigcup_{i=1}^{n} P_{i}=\pi^{-1}(s(\mathcal{F}) \cap S)$ are the projective lines associated to the blow-ups. Let $\tilde{\mathcal{F}}$ be the foliation induced in $\tilde{M}$ and $\tilde{h}=h \circ \pi$ its meromorphic first integral defined in $\tilde{M} \backslash D$. Among $P_{1}, \ldots, P_{n}$ there are perhaps some non-invariant lines. By the previous proposition, $\tilde{h}$ automatically extends to these lines outside their intersection with other invariant lines.

Let $\tilde{D}$ be the set of invariant curves in $D$. We decompose $\tilde{D}=\bigcup_{j=0}^{k} D_{j}$, where each $D_{j}$ is connected and $D_{i} \cap D_{j}=\emptyset$ if $i \neq j$. $D_{0}$ is taken to be the component which contains $P_{0}$. Our job is now reduced to searching separatrices through each $D_{j}$ which are not contained in $D_{j}$. Since we are dealing with generalized curves, this is equivalent to the existence of a singularity of $\tilde{\mathcal{F}}$ outside a corner. Suppose such a separatrix exists at a point $p$ contained in some component $D_{j_{0}}$. Denote by $S_{0}$ the separatrix and by $P_{i_{0}}$ the line which contains $p$. Since $p$ is not a saddle-node and a meromorphic first integral is defined in a neighborhood of $S_{0} \backslash\{p\}$, by applying Extension Lemma 2, it is possible to extend $\tilde{h}$ to a neighborhood of $p$. Lemma 3 allows us to extend $\tilde{h}$ to $P_{i_{0}} \backslash\left\{q_{1}, \ldots, q_{l}\right\}$, the points of intersection of $P_{i_{0}}$ with other lines in $D_{j_{0}}$. Now we apply the same process and extend $\tilde{h}$ to a neighborhood of each $q_{j}$ and, as a consequence, to the lines which contain them. This procedure is repeated until $\tilde{h}$ is extended throughout $D_{j_{0}}$.

Next we show that it is always possible to find a separatrix through $D_{1}, \ldots, D_{n}$. We do not always assure the existence of a separatrix through $D_{0}$. However, some conditions may be given so that this occurs.

Let $M$ be a complex surface and $\mathcal{F}$ a singular holomorphic foliation. The algebraic multiplicity (or simply the multiplicity) of $\mathcal{F}$ at $p \in M$, denoted by $m_{p}(\mathcal{F})$, is the lowest order of the terms appearing in the Taylor series of $\omega_{p}$, some holomorphic one-form which gives the foliation at $p$. Let $S$ be a smooth separatrix through $p$. Choose a local system of coordinates $(x, y)$ at $p$ such that $S=\{y=0\}$ and $\omega_{p}=p(x, y) d x+q(x, y) d y$ is a defining one-form for $\mathcal{F}$. The tangent multiplicity of $\mathcal{F}$ and $S$ at $p, m_{p}(\mathcal{F}, S)$, is the order of $q(x, 0)$ at $x=0$. If $S$ is one of the separatrices of a simple singularity, or the strong separatrix of a saddle node, then $m_{p}(\mathcal{F}, S)=1$. We also have that $p$ is a regular point if and only if $m_{p}(\mathcal{F}, S)=0$.

Let $\pi: \tilde{M} \rightarrow M$ be a sequence of blow-ups starting at $p \in M$ and $D=\pi^{-1}(p)$ the associated divisor. It is proved in [CLS] that

$$
m_{p}(\mathcal{F})+1=\sum_{q \in P \subset D}(\rho(P)) m_{q}^{*}(\mathcal{F}, P),
$$


where

$$
m_{q}^{*}(\mathcal{F}, P)= \begin{cases}m_{q}(\mathcal{F}, P) & \text { if } q \text { is not a corner }, \\ m_{q}(\mathcal{F}, P)-1 & \text { if } q \text { is a corner }\end{cases}
$$

and $\rho(P)$ is a weight associated to $P$. For our purposes, it is sufficient to know that $\rho(P)=1$ when $P$ is associated to the first blow-up.

LEMMA 4. Let $p$ be a singularity of a singular holomorphic foliation $\mathcal{F}$ admitting $a$ smooth separatrix S. Suppose that $p$ is a generalized curve. Then $p$ admits another separatrix distinct from $S$.

PROOF. If $p$ is dicritical, there is nothing to prove. Suppose that $p$ admits a finite number of separatrices. If $p$ is already reduced, then it is simple and has two transversal smooth separatrices. If $p$ is not reduced, we desingularize it and prove by induction in the number of blow-ups.

Suppose first that one blow-up desingularizes $\mathcal{F}$. Denote by $P$ the projective line introduced, by $\tilde{S}$ the strict transform of $S$ (which is smooth and transversal to $P$ ), and set $p_{0}=P \cap \tilde{S}$. If there exists another singularity of $\tilde{\mathcal{F}}$ in $P$, it is reduced and has a separatrix transversal to $P$. So, let us examine the case where $p_{0}$ is a unique singularity in $P$. It is reduced and has $P$ and $\tilde{S}$ as the set of its separatrices. We have

$$
m_{p}(\mathcal{F})+1=m_{p_{0}}(\tilde{\mathcal{F}}, P)=1,
$$

which implies $m_{p_{0}}=0$, an absurdity.

Suppose now that $n>1$ is the number of blow-ups necessary to desingularization and that the result is already proved for singularities which desingularize in less than $n$ steps. Let us perform a first blow-up at $p$, introducing $P, \tilde{S}$ and $p_{0}$ as above. If there exists a singularity $q \in P$, distinct from $p_{0}$, then the induction hypothesis applies to assure the existence of a separatrix through $q$ distinct from $P$. It remains to consider the case where the only singularity in $P$ is $p_{0}$, having $P$ and $\tilde{S}$ as the set of its separatrices. However, according to [CLS], a generalized curve having exactly two transversal smooth separatrices is reduced. The argument of the preceding paragraph applies here to achieve a contradiction.

REMARK 1. Lemma 4 may be false if $S$ is not smooth. For instance, take $p=(0,0) \in$ $C^{2}, S: x^{2}-y^{3}=0$ and $\mathcal{F}: d\left(x^{2}-y^{3}\right)=2 x d x-3 y^{2} d y=0 . p$ is a generalized curve having $S$ as its unique separatrix.

At this point, we are ready to prove Theorem A:

Proof of Theorem A. We suppose that $S$ is $\mathcal{F}$-invariant, since the other case was already proved. Applying Lemma 4 , we extend $h$ to $S \backslash\left\{p_{1}, \ldots, p_{n}\right\}$, where $p_{1}, \ldots, p_{n}$ are the other singularities of $\mathcal{F}$ in $S$. Since these points form a codimension two analytic set, $h$ extends through them, yielding a meromorphic first integral for $\mathcal{F}$ defined in $M$.

Remark that the conclusion of the theorem implies that all singularities of $\mathcal{F}$ in $S$ are generalized curves. 
Let $M$ be a complex surface. Let $S=\bigcup_{i=1}^{n} S_{i} \subset M$ be a finite union of compact complex curves. The matrix $M_{S}=\left(s_{i j}\right)_{1 \leq i, j \leq n}$, where $s_{i j}=S_{i} \cdot S_{j}$, is called the intersection matrix associated to $S$. Notice that $M_{S}$ is symmetric and has real entries.

Observe that if $M_{0} \in M_{n}(\boldsymbol{R})$ is symmetric and $Q \in M_{n}(\boldsymbol{R})$ is non-singular, then $M_{0}$ is negative definite if and only if $Q^{t} M_{0} Q$ is. As a consequence, a permutation of columns followed by the corresponding permutation of lines of a negative definite, symmetric, real matrix yields a negative definite, symmetric, real matrix. This means that the negative definiteness of the intersection matrix of a curve is independent from the enumeration associated to its components. The following is proved in [La]:

THEOREM 1. Let $\pi: \tilde{M} \rightarrow M$ be a sequence of a finite number of blow-ups at $p \in M$ and $D=\pi^{-1}(p), D=\bigcup_{i=1}^{n} P_{i}$, where $P_{i}$ are projective lines. Then the intersection matrix $M_{D}$ is negative definite.

We establish now a connection between the negative definiteness of the intersection matrix $M_{S}$ and the existence of separatrices through a divisor $S$.

Let $S=\bigcup_{i=1}^{n} S_{i}$ be a union of complex curves in a complex surface $M$. To $S$ we associate a graph $\Gamma_{S}$ constructed in the following way: The set of vertices $V_{\Gamma_{S}}=\left\{V_{1}, \ldots, V_{n}\right\}$ corresponds bijectively to the set of components of $S$; to each point in $S_{i} \cap S_{j}$ we define an edge connecting $V_{i}$ and $V_{j}$. We have the following proposition:

PROPOSITION 3 ([C]). Let $M$ be a complex surface with a singular holomorphic foliation $\mathcal{F}$. Let $S=\bigcup_{i=1}^{m} S_{i}$ be a union of $\mathcal{F}$-invariant compact smooth complex curves. Suppose that the singularities of $\mathcal{F}$ in $S$ are non-dicritical and

(i) The associated graph $\Gamma_{S}$ is a tree,

(ii) $M_{S}$ is negative definite.

Then, there exists a separatrix through $S$.

Lemma 5. Let $M_{0} \in M_{n}(\boldsymbol{R})$ be a symmetric negative-definite matrix. If $M_{1} \in$ $M_{n_{1}}(\boldsymbol{R})$ is a submatrix of $M_{0}$ in its diagonal, then $M_{1}$ is negative-definite.

PRoof. We may suppose that $M_{0}$ has the form

$$
M_{0}=\left(\begin{array}{cc}
M_{1} & N^{t} \\
N & M_{2}
\end{array}\right),
$$

where $M_{2} \in M_{n-n_{1}}(\boldsymbol{R})$ and $N \in M_{\left(n-n_{1}\right) \times n_{1}}(\boldsymbol{R})$. If $\mathbf{v} \in \boldsymbol{R}^{n_{1}}, \mathbf{v} \neq 0$, then we have

$$
\mathbf{v} M_{1} \mathbf{v}^{t}=(\mathbf{v}, 0) M_{0}(\mathbf{v}, 0)^{t}<0,
$$

since $M_{0}$ is negative definite. This accomplishes the proof.

Suppose that $M$ carries a singular holomorphic foliation $\mathcal{F}$. Let $\pi$ be a sequence of blow-ups that desingularizes $p \in s(\mathcal{F})$ and $D=\pi^{-1}(p)$ the associated divisor. Denote by $\tilde{D}$ the union of all invariant lines in $D$. Write $\tilde{D}=\bigcup_{i=1}^{n} D_{i}$, where each $D_{i}$ is a connected set coposed by union of projective lines and $D_{i} \cap D_{j}=\emptyset$ if $i \neq j$. We have 
PROPOSITION 4. There exists a separatrix through each $D_{i}$.

PROOF. In fact, after renumbering the projective lines in $D$ if necessary, each $M_{D_{i}}$ will be a submatrix in the diagonal of $M_{D}$. The result follows from the fact that $M_{D}$ is negative definite.

We are at the point of proving Theorem B:

Proof of Theorem B. When $S$ is not $\mathcal{F}$-invariant, the result is already proved. If $S$ is $\mathcal{F}$-invariant, perform the desingularization of $s(\mathcal{F}) \cap S$. Denote by $\pi$ the sequence of blow-ups. Easy calculations show that blowing up a divisor with negative definite intersection matrix yields a divisor with negative definite intersection matrix. The proof of Proposition 4. shows that a divisor contained in a larger divisor with negative definite intersection matrix also has negative definite intersection matrix. Since we depart from a curve $S$ with negative self-intersection number, these facts show that the largest connected set containing $\pi^{*}(S)$ composed by the union of invariant curves of $\pi^{-1}(S)$ has negative definite intersection matrix. This assures that it is crossed by a separatrix. It is therefore possible to extend $h$ to $M$.

We remark that Theorem B may be proved through more general results. A divisor with negative definite intersection matrix may be blown down to a complex surface having normal singularities ([La], Theorem 4.9 and Proposition 4.6). On the other hand, a theorem of Levi assures the extension of a meromorphic function defined outside a codimension-two variety in a normal complex space ([N], Theorem VII-4). The proof we present here has a virtue of relying on properties of foliated surfaces.

In the following lines we make an attempt to extend a meromorphic first integral through a smooth complex curve with non-negative self-intersection number.

Let $p$ be a non-reduced singularity of $\mathcal{F}$ in an invariant curve $S$, which is smooth at $p$. A linear chain at $p$ (with respect to $S$ ) (see [CS]) is a sequence of blow-ups performed in the following way: Let $\pi_{1}$ be a blow-up at $p$ and $P_{1}=\pi_{1}^{-1}(p)$. If $p_{1}=\pi_{1}^{*}(S) \cap P_{1}$ is reduced, then the linear chain at $p_{1}$ is $\pi_{1}$. If $p_{1}$ is non-reduced, then make another blow-up $\pi_{2}$ at $p_{1}$ and, if necessary, successive blow-ups at the corners, until all of them are reduced; the linear chain at $p$ consists of the composition $\pi_{n} \circ \ldots \circ \pi_{1}$ of these blow-ups. We make the following definition:

DEFINITION 1. Let $p$ be a singularity of a germ of holomorphic foliation $\mathcal{F}$ admitting a germ of smooth separatrix $S$. We say that $p$ is an ordinary dicritical singularity if the desingularization of $p$ has one non-invariant projective line lying in the divisor associated to the first linear chain with respect to $S$.

EXAMPLE 1. Let $S_{1}$ and $S_{2}$ be two smooth algebraic curves in $\boldsymbol{C} P^{2}$. Choose an affine plane $C P^{2} \backslash L_{\infty}$ such that $L_{\infty}$ does not intersect $S_{1} \cap S_{2}$. Let $p_{1}(x, y)=0$ and $p_{2}(x, y)=0$ be irreducible polynomial equations for $S_{1}$ and $S_{2}$ in $C P^{2} \backslash L_{\infty}$. Let $\mathcal{F}$ be the foliation in $C P^{2}$ induced by $\omega(x, y)=p_{2}^{2} d\left(p_{1} / p_{2}\right)=p_{1} d p_{2}-p_{2} d p_{1}=0$. Then $S_{1} \cap S_{2}$ is 
composed by dicritical singularities of $\mathcal{F}$ which are ordinary dicritical with respect to both $S_{1}$ and $S_{2}$. We remark that if $S_{1}$ and $S_{2}$ are transversal, then, by Bezout's theorem, $S_{1} \cap S_{2}$ has degree $\left(S_{1}\right)$ degree $\left(S_{2}\right)$ points. In particular, if degree $\left(S_{1}\right)<\operatorname{degree}\left(S_{2}\right)$, then $S_{1}$ contains more than $\left(\operatorname{degree}\left(S_{1}\right)\right)^{2}=S_{1} \cdot S_{1}$ ordinary dicritical singularities.

The above definition explains the statement of Theorem $\mathrm{C}$, which we prove now:

Proof OF THEOREM C. We prove by induction in the intersection number of $S$. Suppose first $S \cdot S=0$. Let $p \in S$ be an ordinary dicritical singularity. If, in the sequence of blow-ups that produces the linear chain from $p$, a dicritical line intersects the strict transform of $S$, then, at this moment, this will have negative self-intersection number. Theorem $\mathrm{C}$ applies to this case. Otherwise, we will reach the following situation: The strict transform $\tilde{S}$ of $S$ will have self-intersection number at most -2 , while the intersection number of the projective line $P$ (intersecting $S$ ) will be -1 . The intersection matrix associated to the divisor $\tilde{S} \cup P$ will clearly be negative definite. Further steps in the desingularization process will take this to a divisor with negative definite intersection matrix.

Suppose now that $S \cdot S=n>0$ and the result is valid for curves with self-intersection number less than $n$. We may suppose that all $n+1$ ordinary dicritical singularities lie in the second case of the previous paragraph. Otherwise we reduce to a curve of smaller intersection number and apply the induction hypothesis. After an appropriate sequence of blow-ups, we reach the situation where $\tilde{S}$ has self-intersection number at most $n-2(n+1)=-n-2$ and $P_{i} \cdot P_{i}=-1$ for $i=1, \ldots, n+1$ (each $P_{i}$ is a projective line intersecting $\tilde{S}$ belonging to the first linear chain of one of the singularities related above). The divisor $D=\tilde{S} \cup P_{1} \cup \ldots \cup P_{n+1}$ has the following $(n+2) \times(n+2)$ intersection matrix

$$
M_{D}=\left(\begin{array}{cccc}
\tilde{S} \cdot \tilde{S} & 1 & \ldots & 1 \\
1 & -1 & \ldots & 0 \\
& & \ldots & \\
1 & 0 & & -1
\end{array}\right)
$$

which is negative definite. This concludes the proof.

3. Some Consequences. We present in this section several situations where Theorems A, B and C apply.

COROLLARY 1. Let $\mathcal{F}$ be a parabolic foliation on $C P^{2}$ whose leaves are proper outside some algebraic invariant curve $S \subset \boldsymbol{C} P^{2}$. Assume that the singularities of $\mathcal{F}$ along $S$ satisfy the hypothesis of Theorem $A, B$ or $C$. Then $\mathcal{F}$ exhibits a rational first integral.

Proof. A theorem of Suzuki ([Su]) implies that $\mathcal{F}$ admits a meromorphic first integral on $\boldsymbol{C} P^{2} \backslash S$, since $S$ is a Stein manifold.

COROLlaRY 2. Let $X$ be a polynomial vector field on $\boldsymbol{C}^{2}$. Suppose that the orbits of $X$ have total finite curvature and are complete for the Euclidean metric on $\boldsymbol{C}^{2}$ (this implies 
that the line at infinity, $l_{\infty}$, is invariant). If there are no affine invariant lines for $X$ and if the singularities of the corresponding projective foliation on $\mathrm{CP}^{2}$ are as in Theorem A, we conclude that $X$ admits a rational first integral and its orbits are contained in algebraic curves.

PROOF. A well-known theorem of Osserman on minimal surfaces assures that each orbit is a parabolic Riemann surface ([W]), so that $\mathcal{F}$ is parabolic. According to [Sc] the fact that the total curvature is finite also implies that the orbits are properly embedded in $\boldsymbol{C}^{2}$. The result then follows from the corollary above.

COROLlary 3. Let $\mathcal{F}$ and $\mathcal{F}_{1}$ be projective foliations on $C P^{2}$. Assume that $\mathcal{F}$ is a pencil by algebraic curves of genus $g \geq 2$, and that there exists some analytic automorphism $T: C^{2} \rightarrow C^{2}$ that conjugates $\mathcal{F}$ and $\mathcal{F}_{1}$ on $\boldsymbol{C}^{2}$. Assume also that the singularities of $\mathcal{F}_{1}$ along the line at infinity are as in Theorem $A$. Then $\mathcal{F}_{1}$ admits a rational first integral and $T$ is algebraic.

Proof. First we observe that $\mathcal{F}_{1}$ admits a meromorphic first integral and therefore a rational first integral by Theorem A. Therefore $T$ is an analytic automorphism of $\boldsymbol{C}^{2}$ that takes algebraic curves into algebraic curves. Since the algebraic curves involved have genus $g \geq 2$ it follows from a result of Kizuka ([K]) that $T$ must be algebraic.

4. Examples. We give some examples where there are obstructions to extend a meromorphic first integral.

EXAMPLE 2. Consider the foliation $\mathcal{F}$ in $\boldsymbol{C} P^{2}$ induced by

$$
\omega=d y-(a(x) y+b(x)) d x=0,
$$

where $a(x)$ and $b(x)$ are polynomials. Let $A(x)$ be a primitive for $a(x)$ and $B(x)$ a primitive for $b(x) / \exp (A(x))$. The meromorphic function

$$
F(x, y)=\frac{y}{\exp (A(x))}-\exp (B(x))
$$

is a first integral for $\mathcal{F}$ in $C P^{2} \backslash L_{\infty}$. All singularities of $\mathcal{F}$ are contained in $L_{\infty}$. We have the following cases:

(i) If degree $(a)<\operatorname{degree}(b)$, then $s(\mathcal{F})$ consists of a single point at $L_{\infty} \cap \overline{\{x=0\}}$. It is a non-reduced singularity, giving rise to a saddle-node by a single blow-up.

(ii) If degree $(a) \geq \operatorname{degree}(b)$, then the crossing $L_{\infty} \cap \overline{\{x=0\}}$ is also a non-reduced singularity, which produces a saddle-node after one blow-up. In this case, $L_{\infty}$ contains another singularity, which is a saddle-node.

The above example does not admit a rational first integral, since it contains saddle-nodes in $L_{\infty}$ (see Proposition 1). 
EXAMPLE 3. The following construction is carried out by means of the techniques of [L]. We construct a surface $M_{0}$ provided with a foliation $\mathcal{F}_{0}$, having an invariant projective line $P_{0}$ such that $P_{0} \cdot P_{0}=-1$, with two singularities $p_{1}$ and $p_{2}$, both of them are linearizable with index $-1 / 2$ with respect to $P_{0}$. We also construct a surface $M_{1}$ provided with a foliation $\mathcal{F}_{1}$, having an invariant projective line $P_{1}$ such that $P_{1} \cdot P_{1}=-1$, with a linearizable singularity $q_{1}$ with index -2 with respect to $P_{1}$, and a second singularity $r_{1}$, which is radial. We define $M_{2}$ to be a copy of $M_{1}$. Similarly, define $\mathcal{F}_{2}$ to be the foliation in $M_{2}, P_{2}$ the invariant projective line, $q_{2}$ and $r_{2}$ the singularities.

We glue a neighborhood of $P_{0}$ in $M_{0}$ with a neighborhood of $P_{1}$ in $M_{1}$ by identifying the local models of $\mathcal{F}_{0}$ in $p_{1}$ and $\mathcal{F}_{1}$ in $q_{1}$, and with a neighborhood of $P_{2}$ in $M_{2}$ by identifying the local models of $\mathcal{F}_{0}$ in $p_{2}$ and $\mathcal{F}_{2}$ in $q_{2}$. The result is a complex surface $M$ with a foliation $\mathcal{F}$ having $P_{0} \cup P_{1} \cup P_{2}$ as an invariant divisor.

Blow up $r_{1}$ and $r_{2}$, giving rise to dicritical lines $\tilde{L}_{1}$ and $\tilde{L}_{2}$. Denote by $\tilde{P}_{0}, \tilde{P}_{1}, \tilde{P}_{2}$ and $\tilde{\mathcal{F}}$ the strict transforms of $P_{0}, P_{1}, P_{2}$ and $\mathcal{F}$, respectively. Choosing a point $s_{1} \in \tilde{L}_{1}$, we provide $\tilde{L}_{1} \backslash\left\{s_{1}\right\}$ with a complex coordinate $z$ such that $\tilde{P}_{1} \cap \tilde{L}_{1}$ corresponds to $z=0$. We define a holomorphic function $H$ in $\tilde{L}_{1} \backslash\{z=0\}$ in the coordinate $z$ by $H(z)=\exp (1 / z)$. $H$ may be extended to a first integral for $\tilde{\mathcal{F}}$ in a neighborhood of $\tilde{L}_{1}$ outside $\tilde{P}_{1}$ by following the leaves of $\tilde{\mathcal{F}}$. Similarly, we extend $H$ to a neighborhood of $\tilde{P}_{1}$ outside $\tilde{P}_{1} \cup \tilde{P}_{0}$ and then to a neighborhood of $\tilde{P}_{0}$ outside $\tilde{P}_{0} \cup \tilde{P}_{1} \cup \tilde{P}_{2}$. Carrying out the same construction starting from $\tilde{L}_{2}$, we will have, by symmetry, a meromorphic first integral $h$ defined in a neighborhood $\tilde{P}_{0} \cup \tilde{P}_{1} \cup \tilde{L}_{1} \cup \tilde{P}_{2} \cup \tilde{L}_{2}$ outside $\tilde{P}_{0} \cup \tilde{P}_{1} \cup \tilde{P}_{2}$. If we blow down $\tilde{L}_{1}, \tilde{P}_{1}$ and $\tilde{L}_{2}, \tilde{P}_{2}$, then the result will be a foliation $\mathcal{G}$ in a complex surface with an invariant line $P$ such that $P \cdot P=1$, having two dicritical singularities and admitting a meromorphic first integral outside $P$. This does not extend to $P$. Notice that these singularities are not ordinary dicritical with respect to $P$, according to our definition. Considering the foliation $\tilde{\mathcal{F}}$ and the complex curve $\tilde{P}_{0} \cup \tilde{P}_{1} \cup \tilde{P}_{2}$, we have an example where theorem A fails when the curve in question is singular.

EXAMPLE 4. Let $\mathrm{G}$ be the group of Möbius maps generated by $g(z)=z /(z+1)$. Let $T$ be a complex torus, $\alpha$ and $\beta$ the generators of $\pi_{1}(T)$ and $\Phi: \pi_{1}(T) \rightarrow G$ the homomorphism such that $\Phi(\alpha)=g, \Phi(\beta)=g$. We make the suspension of this homomorphism, that is, we build a complex fiber bundle $E$ with base $T$ and fiber $\overline{\boldsymbol{C}}$ and a holomorphic foliation $\mathcal{F}$ in $E$ transversal to the fibers such that the holonomy of $\mathcal{F}$ in a fiber is given by $\Phi$ (see [CL]). $\mathcal{F}$ admits a meromorphic first integral in $E \backslash E_{0}$, where $E_{0} \simeq T$ is the null section, constructed in the following way: Let $z$ be a complex coordinate in a fixed fiber $F_{0}$ such that the generator of the holonomy group is written as $g(z)=z /(z+1) . H(z)=\exp (2 \pi i / z)$ is holomorphic outside $\{z=0\}$ and satisfies $H(g(z))=H(z)$ for $z \neq 0$. Therefore, by following the leaves of $\mathcal{F}$, we may extend $H$ to a holomorphic first integral $h$ for $\mathcal{F}$ defined outside $E_{0}$. Of course, $h$ does not extend to $E_{0}$. Notice that the obstruction for the extension is the existence of a map in the holonomy with respect to $E_{0}$ which has the structure of a flower, which implies that its orbits acummulate in the origin (see [C1]). 
EXAMPLE 5. In this example we follow the construction of Riccati foliations with given holonomy, as done in [L]. Let $G$ be the group of Möbius maps generated by $f_{1}(z)=-z$ and $f_{2}(z)=z /(z+j)$, where $j=\exp (2 \pi i / 3)$. $G$ is non-abelian and its generators satisfy $f_{1}^{2}=f_{2}^{3}=\left(f_{1} \circ f_{2}\right)^{6}=$ id. The function $H(z)=\mathcal{P}^{\prime}(1 / z)^{2}$, where $\mathcal{P}$ is the Weierstrass function, is meromorphic in $\overline{\boldsymbol{C}} \backslash\{z=0\}$ and satisfies $H(f(z))=H(z)$ for $f \in G$ (see [F], Section VII-II). We build a fiber bundle $P: E \rightarrow \overline{\boldsymbol{C}}$ with fiber $\overline{\boldsymbol{C}}$ and a singular holomorphic foliation $\mathcal{F}$ in $E$ with three invariant vertical fibers, $F_{0}, F_{1}$ and $F_{2}$, transversal to the fibers in $E \backslash\left(F_{0} \cup F_{1} \cup F_{2}\right)$. Let $E_{0} \simeq \overline{\boldsymbol{C}}$ be the null section. For a fixed fiber $F \neq F_{0}, F_{1}, F_{2}$, with a complex coordinate $z\left(\{z=0\}=F \cap E_{0}\right)$, the holonomy map corresponding to a loop in $E_{0}$ around $p_{1}=P\left(F_{1}\right)$ is given by $f_{1}$, while $f_{2}$ is the holonomy map associated to a loop around $p_{2}=P\left(F_{2}\right)$. The holonomy map associated to a loop around $p_{0}=P\left(F_{0}\right)$ is $\left(f_{1} \circ f_{2}\right)^{-1}$. We obtain a meromorphic first integral $h$ for $\mathcal{F}$ defined outside $E_{0} \cup F_{0} \cup F_{1} \cup F_{2}$ by extending the function $H$ defined in $F \backslash\{z=0\}$ by following the leaves of $\mathcal{F}$. In a neighborhood $V_{i} \times \overline{\boldsymbol{C}}$ of $F_{i}$, with coordinates $\left(x_{i}, z_{i}\right),\left(x_{i}, \hat{z}_{i}\right)$, where $\hat{z}_{i}=1 / z_{i}$ (the fibers correspond to the equations $x_{i}=c$ and $p_{i}$ corresponds to $\left.\left(x_{i}, z_{i}\right)=(0,0)\right), \mathcal{F}$ is given by the equations

$$
\begin{aligned}
& \omega_{i}\left(x, z_{i}\right)=\alpha_{i} z_{i} d x+x_{i} d z_{i}=0, \\
& \hat{\omega}_{i}\left(x, z_{i}\right)=-\alpha_{i} \hat{z}_{i} d x+x_{i} d \hat{z}_{i}=0,
\end{aligned}
$$

where $\alpha_{0}=6, \alpha_{1}=2, \alpha_{2}=3$. Since $i_{p_{i}}\left(\mathcal{F}, E_{0}\right)=-1 / \alpha_{i}$, we have that $c\left(E_{0}\right)=$ $\sum_{i=1}^{3} i_{p_{i}}\left(\mathcal{F}, E_{0}\right)=-1$. It is therefore possible to blow down $E_{0}$ by a map $\pi: E \rightarrow$ $\hat{E} \simeq \boldsymbol{C P} P^{2}$. The foliation $\pi_{*} \mathcal{F}$ has a meromorphic first integral outside the lines $\pi_{*} F_{0}, \pi_{*} F_{1}$ and $\pi_{*} F_{2}$. This does not extend to $C P^{2}$ and the obstruction lies once again in the existence of a map in the holonomy of $\mathcal{F}$ with respect to $E_{0}$ which has a structure of flower (for instance, $\left.\left[f_{1}, f_{2}\right]=f_{1} \circ f_{2} \circ f_{1}^{-1} \circ f_{2}^{-1}=z /(1-2 z)\right)$. Notice that all the singularities of $\pi_{*} \mathcal{F}$ are generalized curves.

5. Foliations in $\boldsymbol{C} P^{2}$. In this section we study foliations in $\boldsymbol{C} P^{2}$ which admit a meromorphic first integral $h$ defined in $C P^{2} \backslash S$, where $S$ is a smooth algebraic curve. We remark that meromorphic functions in $C P^{2}$ are rational, that is, they are given by quotients of polynomial functions. We have the following:

Proposition 5. Let $S$ be an algebraic curve invariant by a foliation $\mathcal{F}$ in $\boldsymbol{C P}^{2}$ with a rational first integral $h$. Then $S$ contains a dicritical singularity.

ProOF. We suppose $h(x, y)=p(x, y) / q(x, y)$, where $p$ and $q$ are non-constant polynomials. Without loss of generality, we may suppose that $S$ is irreducible. Take $\{f(x, y)=0\}$ to be an irreducible polynomial equation defining $S$. The foliation $\mathcal{F}$ is defined by

$$
p(x, y)-\lambda q(x, y)=0, \quad \lambda \in \boldsymbol{C} .
$$

Since $S$ is invariant and irreducible, there exists $\lambda_{0} \in C$ such that $f$ divides $p-\lambda_{0} q$; there exists a polynomial $g$ such that

$$
f(x, y) g(x, y)=p(x, y)-\lambda_{0} q(x, y) .
$$


Substituting (2) in (1), we have the following set of equations:

$$
f(x, y) g(x, y)-\left(\lambda-\lambda_{0}\right) q(x, y)=0, \lambda \in \boldsymbol{C} .
$$

Choose a point $p$ in the intersection of $\{q=0\}$ and $\{f=0\}$. This is a dicritical singularity for $\mathcal{F}$. In fact, assuming that it lies in the affine plane in question (otherwise simply perform an appropriate change of coordinates), (3) gives an infinite number of algebraic curves through $p$.

Let us suppose that a foliation $\mathcal{F}$ in $C P^{2}$ admits a meromorphic first integral in $C P^{2} \backslash S$, where $S$ is a smooth algebraic curve. Theorem A applies to this case if there exists a nondicritical generalized curve in $S$. As a consequence of this theorem and the preceding result, we have

COROLlARY 4. Let $\mathcal{F}$ be a singular holomorphic foliation in $C P^{2}$ admitting a meromorphic first integral outside some smooth algebraic curve $S$. Suppose that a singularity of $\mathcal{F}$ in $S$ is a generalized curve. Then $\mathcal{F}$ has a dicritical singularity in $S$.

PROOF. Let $p \in s(\mathcal{F}) \cap S$ be a generalized curve. If it is non-dicritical, Theorem A says that $\mathcal{F}$ has a rational first integral. Proposition 5 then assures the existence of a dicritical singularity in $S$.

6. Foliations in $C P^{n}$ of codimension 1. Let $\mathcal{F}$ be a codimension one singular holomorphic foliation in $\boldsymbol{C} P^{n}, n \geq 3$. Suppose that $\mathcal{F}$ admits a meromorphic first integral outside some smooth hypersurface $S$. This $n$-dimensional case can be handled by reducing it to a two-dimensional problem.

Let $H \subset C P^{n}$ be an $m$-dimensional complex plane, $2 \leq m \leq n$. We say that $H$ is in general position with respect to $\mathcal{F}$ if $H$ is not $\mathcal{F}$-invariant and $s(\mathcal{F}) \cap H$ is a codimension two analytic set. The proof of the following proposition is adapted from Lemma 5 in [CLS1]:

Proposition 6. Let $\mathcal{F}$ be a singular holomorphic foliation in $\boldsymbol{C P}^{n}$ and $H \subset \mathrm{CP}^{n}$ a hyperplane in general position with $\mathcal{F}$. Then $\mathcal{F}$ admits a rational first integral if and only if $\left.\mathcal{F}\right|_{H}$ does.

PROOF. The "only if" part of the proof is straightforward. Let us prove the opposite implication. It is enough to build a meromorphic first integral for $\mathcal{F}_{V}$, where $V$ is an open neighborhood of $H$. Since $\boldsymbol{C} P^{n} \backslash H$ is a Stein manifold, it extends to $C P^{n}$ ([Siu]). Let $f$ be a meromorphic first integral for $\left.\mathcal{F}\right|_{H}$. Take $p \in H$ a regular point for $\mathcal{F}$. It is possible to find a sufficiently small neighborhood $W_{p}$ of $p$ and a holomorphic coordinate chart $\Psi: W_{p} \rightarrow \Delta$, where $\Delta \subset C^{n}$ is a polydisc, such that:

(i) $\Psi\left(H \cap W_{p}\right)=\left\{z_{n}=0\right\} \cap \Delta$,

(ii) $\Psi_{*}(\mathcal{F})$ is given by $d z_{1}=0$.

Let $\tilde{f}_{p}=\left.f \circ \Psi^{-1}\right|_{\Delta \cap\left\{z_{n}=0\right\}}$. This extends naturally to a meromorphic function defined in $\Delta$, which we still call $\tilde{f}_{p}$, by setting $\tilde{f}_{p}\left(z_{1}, \ldots, z_{n}\right)=\tilde{f}_{p}\left(z_{1}, \ldots, z_{n-1}, 0\right)$. This is a first integral for $\Psi_{*}(\mathcal{F})$. We define $f_{p}=\tilde{f}_{p} \circ \Psi$. 
Notice that, if $W_{p} \cap W_{q} \neq \emptyset, p$ and $q$ being regular points for $\mathcal{F}$, then we have $\left.f_{p}\right|_{W_{p} \cap W_{q}}=\left.f_{q}\right|_{W_{p} \cap W_{q}}$. This follows easily from the identity principle for meromorphic functions. Let $W=\bigcup_{p \in H \backslash s(\mathcal{F})} W_{p} . W$ is a neighborhood of $H \backslash s(\mathcal{F})$, where $\mathcal{F}$ admits a meromorphic first integral, which we call $f_{W}$. All we have to do is extending $f_{W}$ to a neighborhood of $H \cap s(\mathcal{F})$. Since $H$ is in general position with respect to $\mathcal{F}, H \cap s(\mathcal{F})$ is a codimension two analytic set in $H$. Let $p \in H \cap s(\mathcal{F})$. It is possible to find a neighborhood $V_{p}$ of $p$, a change of coordinates $\Phi$ such that $\Phi(p)=0, \Phi\left(V_{p}\right)=\Delta_{1} \times D$ and $\Phi^{-1}\left(\left(\Delta_{1} \backslash \Delta_{2}\right) \times D\right) \subset W \cap V_{p}$, where $\Delta_{2} \subset \Delta_{1} \subset C^{n-1}$ are polydiscs and $D \subset C$ is a disc, all of which centered in the origin. $\left(\Delta_{1} \backslash \Delta_{2}\right) \times D$ is a Hartogs' domain whose holomorphic closure is $\Delta_{1} \times D$. Levi's theorem then allows us to extend $f_{W}$ to $V_{p}$. The result is a meromorphic first integral $F$ defined in $V$, the neighborhood of $H$ consisting of $W \bigcup_{p \in s(\mathcal{F}) \cap H} V_{p}$.

It is proved in [CLS1] that the set of hyperplanes in general position with respect to a foliation $\mathcal{F}$ in $C P^{n}, n \geq 3$, is generic in the set of all hyperplanes.

We can apply the above facts to reduce the extension problem in dimension $n$ to a problem in dimension two. We find a sequence of linear subspaces $H_{2} \subset \ldots \subset H_{n-1} \subset H_{n}=$ $\boldsymbol{C} P^{n}$, where each $H_{i}$ is a linear subspace of dimension $i$, transversal to $H_{i+1} \cap S$, and in general position with respect to $\left.\mathcal{F}\right|_{H_{i+1}}$, for $i=2, \ldots, n-1\left(H_{n}=\boldsymbol{C} P^{n}\right)$. Choosing each $H_{i}$ in such a way that the meromorphic first integral for $\mathcal{F}$ is non-constant over it, $H_{2} \simeq C P^{2}$ will be provided with a foliation $\left.\mathcal{F}\right|_{H_{2}}$ which admits a meromorphic first integral outside $H_{2} \cap S$. Furthermore $\left.\mathcal{F}\right|_{H_{2}}$ admits a rational first integral if and only if $\mathcal{F}$ does.

7. Foliations by curves in higher dimension. Let $M$ be an $n$-dimensional complex manifold with a foliation $\mathcal{F}$ whose leaves are curves $(\mathcal{F}$ is locally induced by a holomorphic vector field). In this section we consider the problem of extending a meromorphic function $F$ defined outside a compact subvariety $S$, whose level surfaces contain the leaves of $\mathcal{F}$. Such a function will still be called a first integral for $\mathcal{F}$. We first remark that if $S$ is of codimension two or greater, $F$ extends meromorphically to $M$ as a consequence of Levi's theorem. Therefore, it is enough to consider the case where $S$ is of codimension one. When $S$ is not $\mathcal{F}$-invariant, the extension is automatic and the proof proceeds as that of Proposition 2:

Proposition 7. Let $M, S, F$ and $\mathcal{F}$ be as above. If $S$ is not $\mathcal{F}$-invariant, then $F$ extends to $M$ as a meromorphic first integral for $\mathcal{F}$.

For the case where $S$ is $\mathcal{F}$-invariant, a higher dimensional version of Extension Lemma 2 is required:

LEMMA 6. Let $F$ be a meromorphic first integral for the linear vector field $X\left(z_{1}, \ldots, z_{n}\right)=\lambda_{1} z_{1} \partial / \partial z_{1}+\cdots+\lambda_{n} z_{n} \partial / \partial z_{n}$, where $\lambda_{i} \neq 0$ for $i=1, \ldots, n$, defined outside the hyperplane $\left\{z_{1}=0\right\}$. If $X$ admits a finite number of separatrices at 0 (outside $\left.\left\{z_{1}=0\right\}\right)$, then $F$ extends to a neighborhood of 0 as a meromorphic first integral for $X$. 
PROOF. We consider the development of $F$ in the Laurent series:

$$
F\left(z_{1}, \ldots, z_{n}\right)=\sum_{i_{1} \in \boldsymbol{Z}, i_{2} \geq l_{2}, \ldots, i_{n} \geq l_{n}} a_{i_{1} \ldots i_{n}} z_{1}^{i_{1}} \ldots z_{n}^{i_{n}} .
$$

Since $F$ is a first integral for $X$ outside $\left\{z_{1}=0\right\}$, we have

$$
\begin{aligned}
0 & =d F\left(z_{1}, \ldots, z_{n}\right) X\left(z_{1}, \ldots, z_{n}\right) \\
& =\sum_{i_{1} \in \mathbf{Z}, i_{2} \geq l_{2}, \ldots, i_{n} \geq l_{n}}\left(\lambda_{1} i_{1}+\cdots+\lambda_{n} i_{n}\right) a_{i_{1} \ldots i_{n}} z_{1}^{i_{1}} \ldots z_{n}^{i_{n}} .
\end{aligned}
$$

Whenever $a_{i_{1} \ldots i_{n}} \neq 0$, we have

$$
\lambda_{1} i_{1}+\cdots+\lambda_{n} i_{n}=0
$$

which is equivalent to

$$
i_{1}=-\frac{\lambda_{2}}{\lambda_{1}} i_{2}-\cdots-\frac{\lambda_{n}}{\lambda_{1}} i_{n} .
$$

Restricting the field $X$ to invariant two dimensional planes $z_{1} \times z_{i}, i=2, \ldots, n$, we see that $\lambda_{i} / \lambda_{1} \in \boldsymbol{Q}$ (since there exists a meromorphic first integral outside $z_{1}=0$ ). On the other hand, the hypothesis on the finite number of separatrices implies that, in fact, $\lambda_{i} / \lambda_{1} \in Q^{+}$. This means that $i_{1}$ is bounded from below by $l_{1}=-\left(\lambda_{2} / \lambda_{1}\right) l_{2}-\cdots-\left(\lambda_{n} / \lambda_{1}\right) l_{n}$, which gives the meromorphic extension of $F$ to the hyperplane $\left\{z_{1}=0\right\}$.

The hypothesis on the number of separatrices is necessary. For instance $F\left(z_{1}, z_{2}, z_{3}\right)=$ $\exp \left(z_{2}^{2} / z_{1}\right)$ is a first integral for $X\left(z_{1}, z_{2}, z_{3}\right)=2 z_{1} \partial / \partial z_{1}+z_{2} \partial / \partial z_{2}+z_{3} \partial / \partial z_{3}$, which does not extend meromorphically to $\left\{z_{1}=0\right\}$. In view of the previous lemma, we may state the following:

THEOREM 2. Let $M, S, F$ and $\mathcal{F}$ be as in the beginning of this section. Assume that $S$ is $\mathcal{F}$-invariant. If $p \in S$ is a linearizable singularity of $\mathcal{F}$, which is a saddle (only non-zero eigenvalues) admitting a finite number of separatrices outside $S$. Then $F$ extends to $M$ as a meromorphic first integral for $\mathcal{F}$.

PROOF. We apply the previous lemma to extend $F$ to a neighborhood of $p$, and Levi's theorem to obtain an extension to the whole $M$.

8. Closed meromorphic one-forms. In this section we seek conditions for extending a closed meromorphic one-form which defines a foliation $\mathcal{F}$ outside a compact complex curve. We remark that in $\boldsymbol{C}^{2}$ closed meromorphic one-forms with simple poles correspond to foliations admitting as a first integral a multiform function of the kind $f_{1}^{\lambda_{1}} \ldots f_{p}^{\lambda_{p}}$, where $f_{1}, \ldots, f_{p}$ are holomorphic and $\lambda_{1}, \ldots, \lambda_{p} \in \boldsymbol{C}$ (see [CM]). We will see that the techniques developed above also apply to this situation. 
PROPOSITION 8. Let $M$ be a complex surface and $S \subset M$ a compact complex curve. Let $\mathcal{F}$ be a singular holomorphic foliation in $M$, which is induced in $M \backslash S$ by a closed meromorphic one-form $\omega$. If $S$ is not $\mathcal{F}$-invariant, then $\omega$ extends to a meromorphic closed one-form in $M$.

PROOF. The proof is similar to that of Proposition 2. Let $p$ be a regular point in $S$, also regular for $\mathcal{F}$, where the foliation is transversal to $S$. Choose $U_{p}$ a coordinate neighborhood around $p$ and $\Phi=(x, y): U_{p} \rightarrow C^{2}$ a coordinate chart such that $P:=\Phi\left(U_{p}\right)$ is a polydisc, $\Phi\left(S \cap U_{p}\right)=\{y=0\}$ and $\left.\mathcal{F}\right|_{U_{p}}$ is the foliation with vertical leaves given by $d x=0$. Let $\tilde{\omega}=\left.\Phi_{*} \omega\right|_{U_{p} \backslash S}$. We have $\tilde{\omega}(x, y)=a(x, y) d x,(x, y) \in P \backslash\{y=0\}$, where $a(x, y)$ is meromorphic in $P \backslash\{y=0\}$. Since $\omega$ is closed, we have that $a(x, y)$ is a function of $x$ only. The extension of $\omega$ to $S$ is achieved by noticing that the singular points of $S$, the tangencies of $\mathcal{F}$ and $S$, and the singularities of $\mathcal{F}$ in $S$ form a codimension two analytic set.

The following is a generalization of Lemma 1:

LEMMA 7. Let $p \in s(\mathcal{F})$ be a simple singularity and $S$ a separatrix for $\mathcal{F}$ at $p$. Suppose that $\mathcal{F}$ is given in a neighborhood $V$ of $S^{*}=S \backslash\{p\}$ by a closed meromorphic one-form $\omega$ with simple poles. Then the holonomy with respect to $S$ is linearizable.

PROOF. Let $\gamma:[0,1] \rightarrow S^{*}$ be a closed path such that $[\gamma] \in H_{1}\left(S^{*}\right)$ is a generator. Choose $\Sigma$ a small disk such that $\gamma \times \Sigma$ is contained in $V$. Suppose first that $S \subset(\omega)_{\infty}$.

Fix $q \in \gamma$. There exists a neighborhood $U$ of $q$ and a local chart $(X, Y)$ in which $\mathcal{F}$ is given by $d Y=0$ and $S \cap U=\{Y=0\}$. Since $\omega$ is closed and has simple poles, it follows that $\omega=a d Y / Y+d \phi$, where $\phi \in \mathcal{O}(U)$ and $a \in C$ is the residue of $\omega$ with respect to $S^{*}$ (hence, independent from $q$ ). From $\omega \wedge d Y=0$, we have $d \phi \wedge d Y=0$, so that $\phi=\phi(Y)$. In a new system of coordinates $(x, y)=(X, Y \exp (\phi(Y))), \mathcal{F}$ is given by $d y=0$, while $\omega=\operatorname{ady} / y$.

It follows that we may cover a neighborhood of $\gamma \times\{0\}$ with a finite number of coordinate charts $\left(x_{j}, y_{j}\right)$ such that $S \cap U_{j}=\left\{y_{j}=0\right\},\left.\mathcal{F}\right|_{U_{j}}: d y_{j}=0$ and $\left.\omega\right|_{U_{j}}=a d y_{j} / y_{j}$. Whenever $U_{i} \cap U_{j} \neq \emptyset$, we have

$$
a \frac{d y_{i}}{y_{i}}=a \frac{d y_{j}}{y_{j}},
$$

so that $y_{i}=c_{i j} y_{j}$, where $c_{i j}$ is locally constant in $U_{i} \cap U_{j}$. It follows that the holonomy mapping associated to $[\gamma]$ is linear.

Suppose now that $S \not \subset(\omega)_{\infty}$. As above, we produce a covering of $\gamma \times\{0\}$ with a finite number of open sets $U_{j}$ provided with coordinates $\left(x_{j}, y_{j}\right)$ such that $\left.\mathcal{F}\right|_{U_{j}}: d y_{j}=0$. We can thus write $\left.\omega\right|_{U_{j}}=a_{j}\left(y_{j}\right) d y_{j}$, where $a_{j}\left(y_{j}\right)$ is holomorphic. Let $A_{j}\left(y_{j}\right)$ be a primitive of $a_{j}\left(y_{j}\right)$ such that $A_{j}(0)=0$. $A_{j}$ is a holomorphic first integral for $\left.\mathcal{F}\right|_{U_{j}}$. If $U_{i} \cap U_{j} \neq \emptyset$, we have $d A_{i}=\left.\omega\right|_{U_{i} \cap U_{j}}=d A_{j}$, which gives $A_{i}=A_{j}$ in $U_{i} \cap U_{j}$. The function $A$ : $U=\bigcup_{j} U_{j} \rightarrow \boldsymbol{C}$ such that $\left.A\right|_{U_{j}}=A_{j}$ is a holomorphic first integral for $\left.\mathcal{F}\right|_{U}$. If $h_{\gamma}$ is the holonomy map associated to $\gamma$, we have that $\left.A\right|_{\Sigma} \circ h_{\gamma}=\left.A\right|_{\sigma}$. Therefore, $h_{\gamma}$ is linearizable. 
LEMma 8 (Extension Lemma I). Let $p \in s(\mathcal{F})$ be a simple singularity and $S$ a separatrix for $\mathcal{F}$ at $p$. Suppose that $\mathcal{F}$ is given in a neighborhood $V$ of $S^{*}=S \backslash\{p\}$ by a closed meromorphic one-form $\omega$ with simple poles. Then $\omega$ extends to a meromorphic one-form defined in a neighborhood of $p$.

PROOF. Lemma 7 and $[\mathrm{MM}]$ give that $\mathcal{F}$ is linearizable at $p$, that is, there are coordinates $(x, y)$ such that the one-form $\eta=x d y-\lambda y d x, \lambda \in \boldsymbol{C} \backslash \boldsymbol{Q}^{+}$, induces the foliation in a neighborhood of $p=(0,0)$. Suppose that $S=\{y=0\}$ in this coordinate system. Let us write

$$
\begin{aligned}
\omega & =a(x, y) d x+b(x, y) d y \\
& =\left(\sum_{j \geq-1, i \in \boldsymbol{Z}} a_{i j} x^{i} y^{j}\right) d x+\left(\sum_{j \geq-1, i \in \boldsymbol{Z}} b_{i j} x^{i} y^{j}\right) d y .
\end{aligned}
$$

Since $\omega$ is closed, we have

$$
\sum_{j \geq-1, i \in \boldsymbol{Z}} i b_{i, j} x^{i-1} y^{j}-\sum_{j \geq-1, i \in \boldsymbol{Z}} j a_{i, j} x^{i} y^{j-1}=0 .
$$

Therefore

$$
(i+1) b_{i+1, j}=(j+1) a_{i, j+1} \text { for } j \geq-1, i \in \boldsymbol{Z} .
$$

On the other hand, since $\omega \wedge \eta=0$ in a neighborhood where both forms are defined, we have

$$
\sum_{j \geq-1, i \in \boldsymbol{Z}} a_{i j} x^{i+1} y^{j}+\lambda \sum_{j \geq-1, i \in \boldsymbol{Z}} b_{i j} x^{i} y^{j+1}=0,
$$

which gives

$$
a_{i, j+1}=-\lambda b_{i+1, j} \quad \text { for } j \geq-1, i \in \boldsymbol{Z} .
$$

Suppose that some $b_{i_{0}, j_{0}} \neq 0$, where $j_{0} \neq-1$. From relations (4) and (5) we have

$$
\lambda=-\frac{a_{i_{0}-1, j_{0}+1}}{b_{i_{0}, j_{0}}}=-\frac{i_{0}}{j_{0}+1}=-\frac{p}{q},
$$

where $p, q \in \boldsymbol{Z}^{+}$are such that $(p, q)=1$. This means that whenever $b_{i, j} \neq 0$ with $j \neq-1$, we have

$$
-\frac{i}{j+1}=-\frac{p}{q} .
$$

That is, there exists $l \in \boldsymbol{Z}$ such that $i=l p$ and $j=-1+l q$. When $b_{i,-1} \neq 0$, equation (4) implies that $i=0$. Therefore the set of indices $(i, j)$ such that $b_{i, j}$ is possibly non-zero is of the form

$$
\left\{\begin{array}{l}
i=l p, \\
j=-1+l q,
\end{array} \quad l \geq 0 .\right.
$$


This means that $b(x, y)$ extends meromorphically to a neighborhood of $p$, possibly having a simple pole in $\{y=0\}$. From equation (5) we see that

$$
\begin{aligned}
& a_{i, j} \neq 0 \Rightarrow b_{i+1, j-1} \neq 0 \\
& \Rightarrow\left\{\begin{array}{l}
i=-1+l p, \quad l \geq 0 . \\
j=l q,
\end{array}\right.
\end{aligned}
$$

Therefore $a(x, y)$ also extends meromorphically to $p$.

In the case of closed forms with poles of higher order we have:

LEMMA 9 (Extension Lemma II). Let $p \in s(\mathcal{F})$ be a simple singularity and $S$ a separatrix for $\mathcal{F}$ at $p$. Suppose that $\mathcal{F}$ is given in a neighborhood $V$ of $S^{*}=S \backslash\{p\}$ by a closed meromorphic one-form $\omega$ with a pole of order $k+1 \geq 2$ in $S$. Then $\omega$ extends to a meromorphic one-form defined in a neighborhood of $p$.

PROOF. If the holonomy of $S$ at $p$ is linearizable, then the proof goes as that of Lemma 8. We therefore suppose that the holonomy is not linearizable. We first remark (see [LSc]) that since $S$ is a pole of order $k+1 \geq 2$ of the closed form $\omega$, the holonomy group of $S$ is conjugated to a subgroup of $G_{k, \lambda}$ for some $\lambda$ in $C$, where

$$
G_{k, \lambda}=\left\{R_{\theta} \circ g_{z, k, \lambda} ; z \in C, \lambda^{k}=1\right\},
$$

and

$$
g_{z, k, \lambda}=\exp \left(z \frac{x^{k+1}}{1+\lambda x^{k}} \frac{\partial}{\partial x}\right) .
$$

It follows from formal calculations that $p$ must be a resonance. We then have at $p$ the following Martinet-Ramis normal form ([MaR, p. 597]): There are formal coordinates at $p$ such that $\mathcal{F}$ is given in a unique way by a form of the model

$$
\omega_{p / q, k, \lambda}=p\left(1+(\lambda-1)\left(x^{p} y^{q}\right)^{k}\right) y d x+q\left(1+\lambda\left(x^{p} y^{q}\right)^{k}\right) x d y,
$$

where $(p, q)=1$. The holonomy maps at $\{y=0\}$ and $\{x=0\}$ are given respectively by

$$
\exp (-2 \pi i p / q) \circ g_{2 \pi i, q k, \lambda q / p}
$$

and

$$
\exp (-2 \pi i q / p) \circ g_{2 \pi i, p k,(\lambda-1) p / q}
$$

Since each germ of diffeomorphism in $(\boldsymbol{C}, 0)$ tangent to the identity is formally conjugated to a unique model $g_{z, k, \lambda}$ ([MaR, p. 580]), we see that the holonomy of $S$ at $p$ is analytically 
normalizable, that is, the coordinates in question are holomorphic. Therefore the MartinetRamis normal form is in fact holomorphic.

On the other hand, $\omega_{p / q, k, \lambda}$ has $h(x, y)=p q x y\left(x^{p} y^{q}\right)^{k}$ as an integrating factor. That is, $\bar{\omega}_{p / q, k, \lambda}=h(x, y)^{-1} \omega_{p / q, k, \lambda}$ is closed. Therefore, there exists a meromorphic function $g$ defined in $V$ such that $\omega=g \bar{\omega}_{p / q, k, \lambda}$. If $g$ were non-constant, it would be a first integral for $\mathcal{F}$ in $V$, since $\omega$ and $\bar{\omega}_{p / q, k, \lambda}$ are closed. Then the holonomy of $S$ at $p$ would be linearizable, which is not the case. Therefore, $g$ is constant and $\omega$ extends to a neighborhood of $p$ as $g \tilde{\omega}_{p / q, k, \lambda}$. This completes the proof.

We also have:

LEMMA 10. Let $M$ be a complex surface and $S$ a compact connected complex curve. Suppose that $\omega$ is a meromorphic one form defined in $M \backslash S$. If $\omega$ extends as a meromorphic one form to $(M \backslash S) \cup V_{p}$, where $V_{p}$ is a neighborhood of a point $p \in S$, then it extends meromorphically to $M$.

PROOF. The proof is similar to that of Lemma 3, noticing that a meromorphic oneform defined in a Hartogs' domain extends to its holomorphic closure.

The proofs of theorems $\mathrm{A}^{\prime}, \mathrm{B}^{\prime}$ and $\mathrm{C}^{\prime}$, stated below, proceed as those of their counterparts, Theorems A, B and C.

THEOREM $\mathrm{A}^{\prime}$. Let $\mathcal{F}$ be a singular holomorphic foliation in a complex surface $M$ induced by a closed meromorphic one-form in $M \backslash S$, where $S$ is a compact, smooth, connected complex curve. If some singularity of $\mathcal{F}$ in $S$ is a non-dicritical generalized curve, then $\omega$ extends to a closed meromorphic one-form in $M$.

THEOREM B'. Let $\mathcal{F}$ be a singular holomorphic foliation in a complex surface $M$ induced by a closed meromorphic one-form in $M \backslash S$, where $S$ is a compact, smooth, connected complex curve with negative self-intersection number. If all singularities of $\mathcal{F}$ in $S$ are generalized curves, then $\omega$ extends to a closed meromorphic one-form defined in $M$.

THEOREM $\mathrm{C}^{\prime}$. Let $\mathcal{F}$ be a singular holomorphic foliation in a complex surface $M$ induced by a closed meromorphic one-form $\omega$ outside a compact, smooth, connected complex curve $S$ with self-intersection number $n \geq 0$. Suppose that the singularities of $\mathcal{F}$ in $S$ are generalized curves. If there are at least $n+1$ ordinary dicritical singularities in $S$, then $\omega$ extends to a closed meromorphic one-form defined in $M$.

\section{REFERENCES}

[C] C. CАмасно, Quadratic forms and holomorphic foliations on singular surfaces, Math. Ann. 282 (1988), $177-184$.

[C1] C. CAMACHO, On the local structure of conformal mappings and holomorphic vector fields in $\boldsymbol{C}^{2}$, Journéss Sirgulières de Dijon (Univ. Dijon, Dijon, 1978), 83-94, Astérisque 60, Société Mathématique de France, Paris, 1978.

[CLS] C. CAmacho,A. Lins Neto And P. SAD, Topological invariants and equidesingularization for holomorphic vector fields, J. Differential Geom. 20 (1984), 143-174. 
[CLS1] C. CAmacho, A. Lins Neto And P. SAD, Foliations with algebraic limit sets, Ann.of Math.136 (1992), 429-446.

[CL] C. CAMACHO AND A. Lins Neto, Geometric theory of foliations, Birkhauser, Boston, 1985.

[CS] C. CAMACHO AND P. SAD, Invariant varieties through singularities of holomorphic vector fields, Ann. of Math. 115 (1982), 579-595.

[CS1] C. CAMACHO AND P. SAD, Pontos singulares de equações diferenciais analíticas, 16 Colóquio Brasileiro de Matematica, Instituto de Matemática Pura e Aplicada, Rio de Janeiro, 1987.

[CM] D. Cerveau and J-F. Mattei, Formes intégrables holomorphes singulières, Astérisque 97, Société Mathématique de France, Paris, 1982.

[F] L. FORD, Automorphic functions, Chelsea Publ. Co., New York, 1951.

[GH] P. GRIFFITHS AND J. HARRIS, Principles of algebraic Geometry, John Wiley, New York, 1994.

[Gun] R. GunNing, Introduction to holomorphic functions of several variables, Vol II, Local theory, Wadsworth \& Brooks/Cole Math. Ser., Pacific Grove, 1990.

[K] T. KizUKA, Analytic automorphisms and algebraic automorphisms of $\boldsymbol{C}^{2}$, Tôhoku Math. J. 31 (1979), $553-565$.

[La] H. B.LAUfer, Normal two-dimensional singularities, Princeton Univ. Press, Princeton, 1971.

[L] A. Lins Neto, Construction of singular holomorphic vector fields and foliations in dimension two, J. Differential Geom. 26 (1987), 1-31.

[LSc] A. Lins Neto AND B. AZEvedo SCÁRduA, Folheações algébricas complexas, 21 Colóquio Brasileiro de Matemática, Instituto de Matemática Pura e Aplicada, Rio de Janeiro, 1997.

[MM] J-F. Mattei and R. Moussu, Holonomie et intégrales prémières, Ann. Sci. École Norm. Sup. (4) 13 (1980), 469-523.

[MaR] J.MARTINET AND RAMIS, J-P., Classification analytique des équations différentielles non linéaires résonnantes du premier ordre, Ann. Sci. École Norm. Sup. (4) 16, 571-621.

[N] R. NARASIMHAN, Introduction to the theory of analytic spaces, Lecture Notes in Math. 25, SpringerVerlage, Berlin-New York, 1966.

[Sc] B. AZEvedo SCÁRDUA, Complex vector fields having orbits with bounded geometry, IMPA, 1998, Preprint.

[Sei] A. SeIdenberg, Reduction of singularities of the differential equation $A d y=B d x$, Amer. J. Math. 90 (1968), 248-269.

[Siu] Y-T. SiU, Techniques of extension of analytic objects, Marcel Dekker, New York, 1974.

[Su] M. SuZUKI, Sur les opérations holomorphes de $\boldsymbol{C}$ et de $\boldsymbol{C}^{*}$ sur un space de Stein, Fonctions de plusieurs variables complexes, III (Sém. François Norguet, 1975-1977), 80-88, 394, Lecture Notes in Math. 670, Springer-Verlag, Berlin, 1978.

[W] B. White, Complete surfaces of finite total curvature, J. Differential Geom. 26 (1987), 315-226.

DEPARTAMENTO DE MATEMÁTICA-ICEX

Universidade Federal de Minas Gerais

AV. ANTÔNIO CARLOS, 6627

30123-970 BELO HORIZONTE MG

BRAZIL

E-mail address: rsmol@mat.ufmg.br 